\section{Empleo del método estratigráfico en el estudio de la vivienda rural vernácula construida con tierra. Un caso de aplicación en La Rioja, Argentina}

\section{The stratigraphic method applied to the assessment of vernacular earthern houses. A particular case study in La Rioja province, Argentina}

Guillermo Rolón*

Rodolfo Rotondaro

Instituto de Arte Americano e Investigaciones Estéticas «Mario J. Buschiazzo» (IAA) Facultad de Arquitectura, Diseño y Urbanismo - Universidad de Buenos Aires

\section{Resumen}

La Arquitectura en Tierra, como campo temático específico dentro de la disciplina de Arquitectura, renace a partir del último cuarto de siglo XX impulsada por la urgente necesidad de preservar este tipo de patrimonio construido en todo el mundo. En la actualidad, su campo de acción se ha incrementado notablemente, del mismo modo que su vinculación con otras disciplinas. En este texto exponemos los resultados previos surgidos del estudio de un edificio histórico doméstico en tierra empleando herramientas teóricas y metodológicas desarrolladas en el ámbito de la Arqueología de la Arquitectura. El caso abordado corresponde a una vivienda rural popular construida durante la segunda mitad del siglo XIX en la región de los valles de la provincia argentina de La Rioja, con continuidad de uso hasta la actualidad. El estudio fue realizado empleando conjuntamente el método estratigráfico de alzados, la tipología de vivienda rural y el análisis de las diferentes técnicas constructivas.

Palabras claves: Arquitectura vernácula, tipología de vivienda, técnicas constructivas en tierra, adobe, Argentina.

\begin{abstract}
Earthen architecture, as being study subject, began in the last quarter of the twentieth century caused by the urgent need to preserve this type of building heritage worldwide. Nowadays, its scope has significantly increased and linked with other disciplinary fields. In this article, we present the preliminary results obtained from the study of earthen historic building by using theoretical and methodological tools developed within Archaeology of Architecture. The case herein discussed is a rural dwelling which was continuously inhabited. It was built during the second half of the nineteenth century in the valley regions in La Rioja province (Argentina). The study was jointly carried out using the wall stratigraphic method, the typology of rural housing and the analysis of different construction techniques.
\end{abstract}

Keywords: Vernacular architecture, housing typology, earthen construction techniques, adobe, Argentina.

\section{INTRODUCCIÓN}

Uno de los proyectos del Programa ARCONTI ${ }^{1}$ del IAA, desde el año 2008, es el estudio de los tipos constructivos y espaciales de la vivienda rural popular con especial énfasis en la provincia argentina de La Rioja y en casos que implican el empleo de la tierra como material de construcción predominante. Esta línea temática surge con el interés en ampliar el conocimiento sobre el patrimonio construido en tierra en el territorio argentino. El objetivo principal de este trabajo es indagar las posibilidades que abre la Arqueología de la Arquitectura (AA), en general, y la lectura estratigráfica de paramentos y la tipología, en particular, al estudio del patrimonio rural construido en tierra. El trabajo utiliza aspectos teóricos y metodológicos propios de la AA con el interés de enriquecer las formas convencionales de investigación de la vivienda rural y el patrimonio construido en tierra. En la primera parte del artículo se presenta un breve contexto del camino que ha recorrido hasta la actualidad el estudio de la Arquitectura en Tierra en Iberoamérica, principalmente en Argentina. Luego se expone la experiencia de un caso analizado a partir del empleo conjunto de la estratigrafía "sobre cota cero" y del estudio tipológico de la vivienda rural argentina y sus técnicas constructivas.

\subsection{La Arquitectura en tierra}

La tierra ha sido uno de los materiales naturales empleados en la construcción del hábitat del hombre durante gran parte de su historia (Bardou y Arzoumanian, 1979; Fathy, 1973; Maldonado Ramos y Rivera Gámez, 2002; Santos Pinheiro, 1993). Actualmente, y a pesar de que los materiales industrializados monopolizan el mercado de la construcción en los centros urbanos y en los ámbitos rurales de gran parte del mundo, la tierra sigue siendo un material utilizado en una variedad importante de edificaciones. Alrededor de un tercio de la población mundial construye $\mathrm{o}$ vive en viviendas donde esta materia prima ha sido aprovechada (Dethier, 1982; Huben y Guillaud, 1994; Rotondaro, 2007). En parte, porque en muchas regiones deben servirse de materiales naturales para construir, condicionados por cuestiones económicas o de imposibilidad de acceso a otros recursos, pero también por razones ideológicas o culturales. La crisis energética del último cuarto del siglo XX contribuyó a revalorizar y extender su empleo debido al reducido consumo de energía que se requiere en el uso de este material (Morel et al, 2001;

${ }^{1}$ Programa de Investigación Arquitectura y Construcción con Tierra, FADU UBA. Director: Mgter. Arq. Rodolfo Rotondaro. 
Tiwari et al, 1995). Progresivamente, el estudio de la Arquitectura en Tierra fue ampliándose con el desarrollo de materiales y técnicas constructivas tanto para el empleo en construcciones nuevas como para su aprovechamiento en la conservación y restauración del patrimonio construido (Jiménez Delgado y Cañas Guerrero, 2007; Maldonado Ramos et al, 2002; Martínez-Camacho et al, 2008; Viñuales, 1981).

\subsection{Los ámbitos de investigación y difusión en Iberoamérica y Argentina}

Entre las décadas de los 70 y 80, la UNESCO, el ICOMOS y el ICCROM realizaron en Asia y América del Sur una serie de encuentros entre profesionales en torno al patrimonio construido en tierra ${ }^{2}$. A partir de estos encuentros de nivel internacional, el tema del Patrimonio de Arquitectura en Tierra comienza a tener mayor reconocimiento en Iberoamérica y a poner en relevancia la tarea que muchos profesionales y constructores venían desarrollando individualmente en torno a él (Viñuales, 1987). Estos acontecimientos no fueron sucesos aislados; acciones de igual índole tuvieron lugar en muchas partes del mundo ante la preocupación por gestionar y preservar este tipo de patrimonio, fomentando su investigación e intensiva formación en este tema ${ }^{3}$.

En 1991, dentro del Subprograma XIV-Viviendas de Interés Social del CYTED (Programa Iberoamericano de Ciencia y Tecnología para el Desarrollo) se desarrolla la Red Temática XIV.A Habiterra (Calla García, 1999). En continuidad con esta Red, en 2001 se aprueba el Proyecto Competitivo XIV.6: PROTERRA, que surge «...con el objetivo de incentivar el uso de la tierra como material de construcción a través de la realización de proyectos demostrativos, publicaciones, cursos y otros eventos» ${ }^{4}$. Los SIACOT (Seminarios Iberoamericanos de Construcción con Tierra) nacen en el seno de esta red y funcionan como principales promotores del intercambio de experiencias en materia de Arquitectura en Tierra. Desde el proyecto PROTERRA el debate ha llevado a establecer que el

\footnotetext{
${ }^{2}$ En 1972 se realiza en Irán la primera Conferencia Internacional en Conservación de Arquitectura en Tierra. Luego en 1976 se realizan los cursos de Restauración como parte del Proyecto Regional de Patrimonio Cultural y Desarrollo y en 1983 se desarrolla el cuarto Simposio Internacional y Curso Taller sobre Conservación del Adobe, ambos en Perú.

${ }^{3}$ En 1987 en Roma, se establece entre ICCROM (Centro Internacional para el Estudio de Conservación y Restauración de Bienes Culturales) y CRATerreEAG (Centro Internacional de Construcción con Tierra de la Escuela de Arquitectura de Grenoble, Francia) el Proyecto Gaia, desde el cual se elaboró un total de seis encuentros formativos conocidos como cursos «PAT» (Preservación de Arquitectura en Tierra).

${ }^{4}$ Declaración de la Red PROTERRA, en www.redproterra.org.
}

concepto de Arquitectura en Tierra es «el conjunto de todas la manifestaciones constructivas, arquitectónicas y urbanísticas que han sido proyectadas y construidas con la tierra como material predominante» (Neves, 2004), idea empleada en este trabajo.

En Argentina, el estudio de la Arquitectura en Tierra tiene antecedentes claros en diversos trabajos desarrollados durante la primera mitad del siglo XX que tratan sobre la vivienda natural, la vivienda rural, la vivienda vernácula y el rancho (Aparicio, 1931 y 1937; Ardissone, 1948; Chiozza, 1945; Zamorano, 1950). Como fruto de estos antecedentes y de nuevos estudios, se publicó posteriormente un tratado general en relación a la «vivienda natural ${ }^{6}$ que abarcó todo el territorio argentino (FAU, 1969). Este trabajo se realizó con un enfoque exclusivamente arquitectónico. En él se documentó una importante variedad de tipologías y técnicas constructivas de la arquitectura vernácula en tierra de las distintas regiones del país. Otros trabajos posteriores, circunscriptos específicamente a la provincia de La Rioja (Armellini et al, 1970; Canepuccia et al, 1976), abordaron el tema de «la vivienda tradicional» en zonas áridas. Estos trabajos expusieron un análisis del diseño de la arquitectura en tierra en la región de valles y quebradas de esta provincia con el objeto de establecer propuestas adecuadas al medio. Sin embargo, en estos casos, se hizo hincapié únicamente en aspectos de diseño arquitectónico.

Los primeros artículos en tratar plenamente el tema de la construcción con tierra en Argentina comenzaron a aparecer durante la década de los ochenta con principal atención en el estudio de las técnicas constructivas tradicionales y en la conservación y preservación de este tipo de patrimonio arquitectónico (AA.VV., 1987; Viñuales, 1981, 1990 y 1991; Viñuales et al, 1994). Luego, con un cuerpo más amplio de profesionales, investigadores y constructores involucrados en esta cuestión, la actividad experimentó un proceso de ampliación de temas y trabajos que profundizaron en nuevos aspectos como la problemática de la vivienda de interés social, el desarrollo tecnológico, la transferencia de conocimientos, la vinculación entre patrimonio y turismo y la intervención sobre

\footnotetext{
${ }^{5}$ Nombre común empleado para designar la vivienda rural popular en Argentina.

${ }^{6}$ Se consideraba como vivienda natural aquella que estaba: 1 . Construida por sus propios moradores y con predominio de materiales de la zona. 2. Organizada en base a módulos o unidades de planta cuadrangular o rectangular, de tipo semiindependiente y repetitivo, en sentido horizontal. 3. Alojaba grupos familiares cuyos medios de subsistencia dependen de actividades primarias. Diferenciándose así del concepto hasta entonces ambiguo y ampliamente difundido de «rancho» (FAU, 1969).
} 

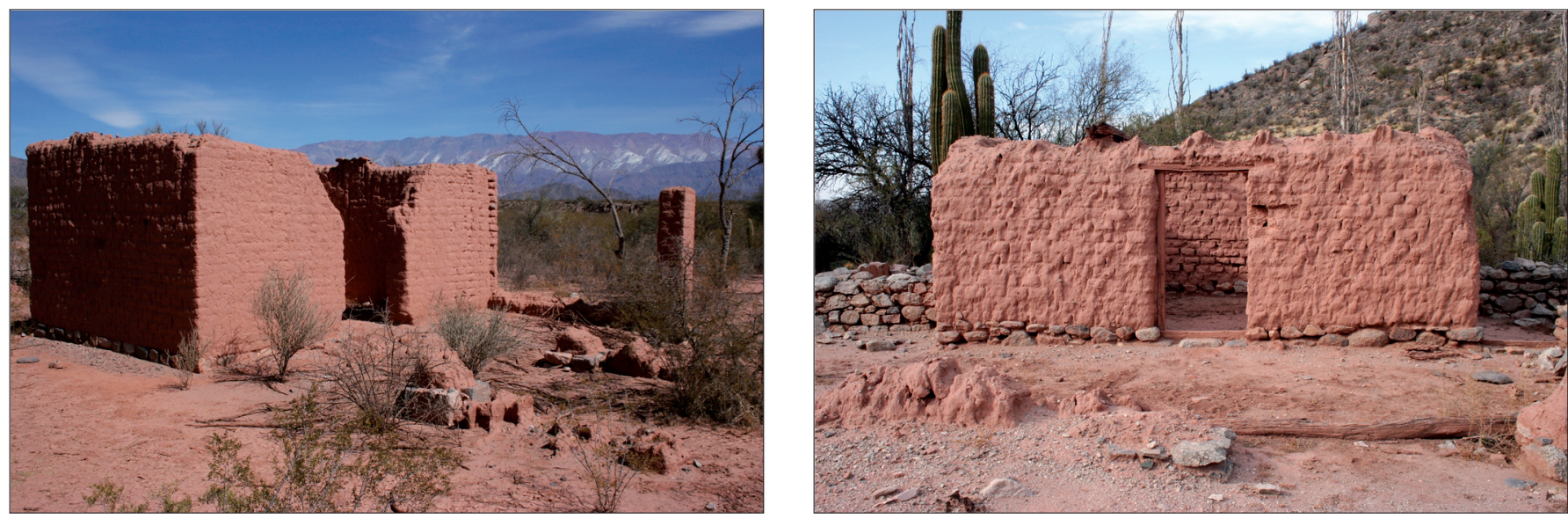

Fig. 1. Dos ejemplos de viviendas con la unidad de habitación original. Ambas permanecen en estado de abandono. Chañarmuyo, La Rioja. Fotografía colección personal de los autores

el patrimonio construido en tierra. Durante este proceso se consolidaron diversos grupos y se creó el centro CRIATiC (Centro Regional de Investigación de Arquitectura de Tierra Cruda), dependiente de la Universidad Nacional de Tucumán.

\section{LA VIVIENDA RURAL EN TIERRA EN LA RIOJA ARGENTINA}

La tierra fue el material de uso predominante en gran parte de la arquitectura de la provincia argentina de La Rioja, hasta aproximadamente los últimos 50 años, con un empleo significativo de la albañilería de adobe y los techos de torta ${ }^{7}$ de una sola pendiente o completamente planos. $\mathrm{Su}$ estudio fue abordado en numerosas investigaciones (Aparicio, 1937; Armellini et al, 1970; Cáceres Freyre, 1946; Canepuccia et al, 1976; Viñuales, 1981; Rotondaro et al, 1993). En las últimas décadas, una mejor comunicación con los centros de servicios y la disponibilidad de nuevos materiales industrializados introdujeron cambios de las técnicas constructivas. Cirvini (2005) plantea que este y otros factores ${ }^{8}$ han provocando un abandono gradual de las prácticas constructivas en tierra, algo que es posible observar en varias partes de la región del centro oeste argentino, principalmente en aquellas zonas mejor vinculadas a centros urbanos. Por su parte, ICOMOS ya advertía desde fines del siglo XX sobre la vulnerabilidad de estas técnicas constructivas frente a la homogeneización que

\footnotetext{
${ }^{7}$ Torta: Capa de barro que se coloca sobre un entramado para formar la cubierta (Viñuales et al, 1994: 125 y De Hoz Onrubia et al., 2003: 197).

${ }^{8}$ Considera además, el desprestigio en el plano simbólico, la marginación de ámbitos académicos y científicos, la escasez de recursos humanos artesanales, técnicos y profesionales y la falta de reglamentación o incluso la prohibición de su empleo en determinadas jurisdicciones.
}

plantea el empleo de materiales de procedencia industrial ${ }^{9}$. Sin embargo, es en el ámbito rural donde aún es posible encontrar vigentes parte de las características de esta tradición constructiva vernácula.

En el área rural de La Rioja observamos que resulta común el abandono de la vivienda, su uso ininterrumpido y en menor medida la reutilización o cambio de función. Las viviendas rurales presentan un crecimiento casi exclusivamente en planta y es característica la adición por yuxtaposición de nuevas construcciones conformando alineaciones o bien ángulos rectos. En la gran mayoría de los casos observados, la unidad que da origen a estas viviendas está formada por una habitación de entre quince a veinte metros cuadrados con una galería en uno de sus laterales (al que denominaremos en adelante «unidad original»). El espacio principal en estas viviendas suele ser la galería, sector que se orienta de forma preferencial hacia el Norte para aprovechar el asoleamiento y la protección de los vientos. En un porcentaje importante, las primeras viviendas del poblado de Chañarmuyo, departamento de Famatina, contienen en el conjunto del edificio esta unidad habitacional indicada (Fig. 1). Las construcciones suelen tener muros de espesores importantes (mayores a $20 \mathrm{~cm}$ ) y pocas

\footnotetext{
9 «Si el Patrimonio Vernáculo construido constituye el modo natural y tradicional en que las comunidades han producido su propio hábitat, la arquitectura en tierra representa el más amplio espectro de variantes de este rico patrimonio en el planeta. Este vasto conjunto de obras padecen procesos de transformación en una continua adaptación como respuesta a los requerimientos sociales y ambientales. La continuidad de esa tradición se ve amenazada en todo el mundo por las fuerzas de la homogeneización cultural y arquitectónica y por la globalización socio-económica. De allí que tanto las estructuras como las técnicas vernáculas son, en todo el mundo, extremadamente vulnerables y se enfrentan a serios problemas de obsolescencia, equilibrio interno e integración.» Carta del Patrimonio Vernáculo Construido ICOMOS - México 1999.
} 


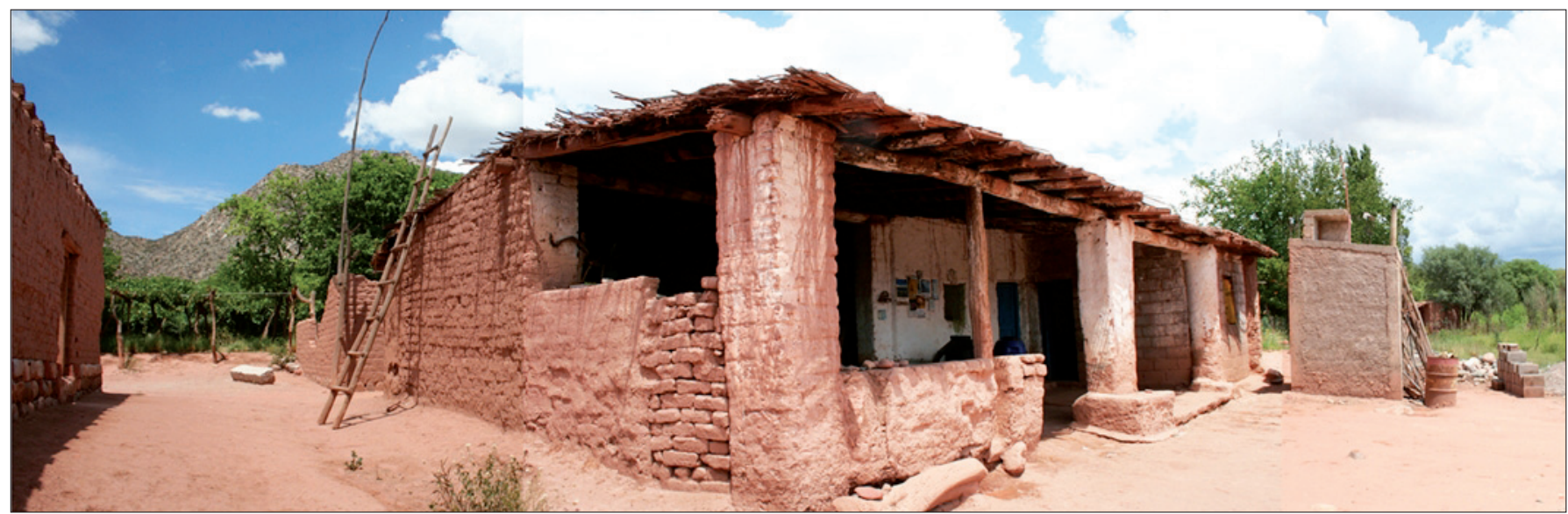

Fig. 2. Vivienda de estudio. Casa de Zulma Castro, Chañarmuyo, La Rioja. Fotografía colección personal de los autores

y pequeñas aberturas para evitar las pérdidas o ganancias de calor, ocasionadas por la alta amplitud térmica diaria.

\subsection{Casa Zulma Castro}

El estudio de caso corresponde a una vivienda rural popular con continuidad de uso hasta la fecha, ubicada en el sector original del pueblo de Chañarmuyo (Fig. 2). Esta construcción se encuentra en una región caracterizada por su clima semiárido de tipo continental, característico del centro oeste argentino.

Éste es un caso destacado de Arquitectura en Tierra en la zona por su antigüedad, su estado de conservación, por presentar numerosas etapas constructivas (producto de su uso ininterrumpido) y donde la identificación de la totalidad de las técnicas constructivas no presentaba mayores inconvenientes.

\subsection{Secuencia estratigráfica}

Para poder determinar las aparentes secuencias constructivas de la vivienda, fue necesario trabajar en conjunto diversos instrumentos de análisis como la estratigrafía de alzados (Parenti, 1996), las técnicas constructivas y la tipología de la vivienda rural. A partir de este análisis se determinó la existencia de al menos cinco fases constructivas, identificándose cada fase con instancias de ampliación de la vivienda. Los relatos de sus ocupantes permitieron estimar la antigüedad de la construcción entre unos 100 a 150 años. En este sentido, la individualización del tipo espacial de la unidad original contenido en la estructura general de la vivienda corroboró dicha afirmación (Figs. 3 y 4, Fase I).

\section{Fase I}

Corresponde a la primera construcción de la vivienda rural conformada por una habitación con pocas y pequeñas aberturas y una galería sobre el lado más largo orientada al Noroeste. El material de tierra se empleó en prácticamente toda la construcción. Los muros se componen de adobes de $40 \times 20 \times 10 \mathrm{~cm}$ colocados a tizón, obteniendo de este modo muros de $40 \mathrm{~cm}$ de espesor nominal. Las columnas son circulares, utilizándose adobes semicirculares de $40 \times 10 \times 20 \mathrm{~cm}$ de radio. Las paredes interiores fueron revocadas con mortero de tierra; exteriormente, sólo la que se encuentra protegida por la galería conserva el revoque. El techo está formado por una «torta pesada» ${ }^{10}$ compuesta por varias capas entre las que se observó: un cielorraso de cañas atadas regularmente perpendiculares a la pendiente del techo, una enramada ${ }^{11}$, una capa de tela arpillera y finalmente la cubierta de torta. En la estructura del techo se emplearon rollizos de madera de algarrobo sin desbastar (Prosopis spp.) en sentido paralelo a la pendiente. La habitación cumplió la función de dormitorio y de espacio para el guardado de las pertenencias personales, mientras que la galería fue un espacio semicubierto multiuso, permitiendo desarrollar, como es habitual para este tipo de espacios y para esta región geográfica, gran parte de las actividades diurnas.

\section{Fase II}

Es la primera ampliación de la vivienda. Estableció un espacio semicerrado más amplio y específico para las actividades diarias sobre el lateral noreste de la unidad

\footnotetext{
${ }^{10}$ Torta pesada: Viñuales (1981: 11) diferencia entre torta pesada y torta liviana por la presencia o no respectivamente de un entramado de ramas entre las capas del techo.

${ }^{11}$ Capa inferior de la cubierta que sirve de soporte para las capas superiores. Por lo general, está formada por un entramado de ramas secas y planas. Se emplean diversas plantas autóctonas, principalmente ramas de Larrea divaricata y $L$. cuneifolia, abundantes en la región fitogeográfica argentina de Monte.
} 

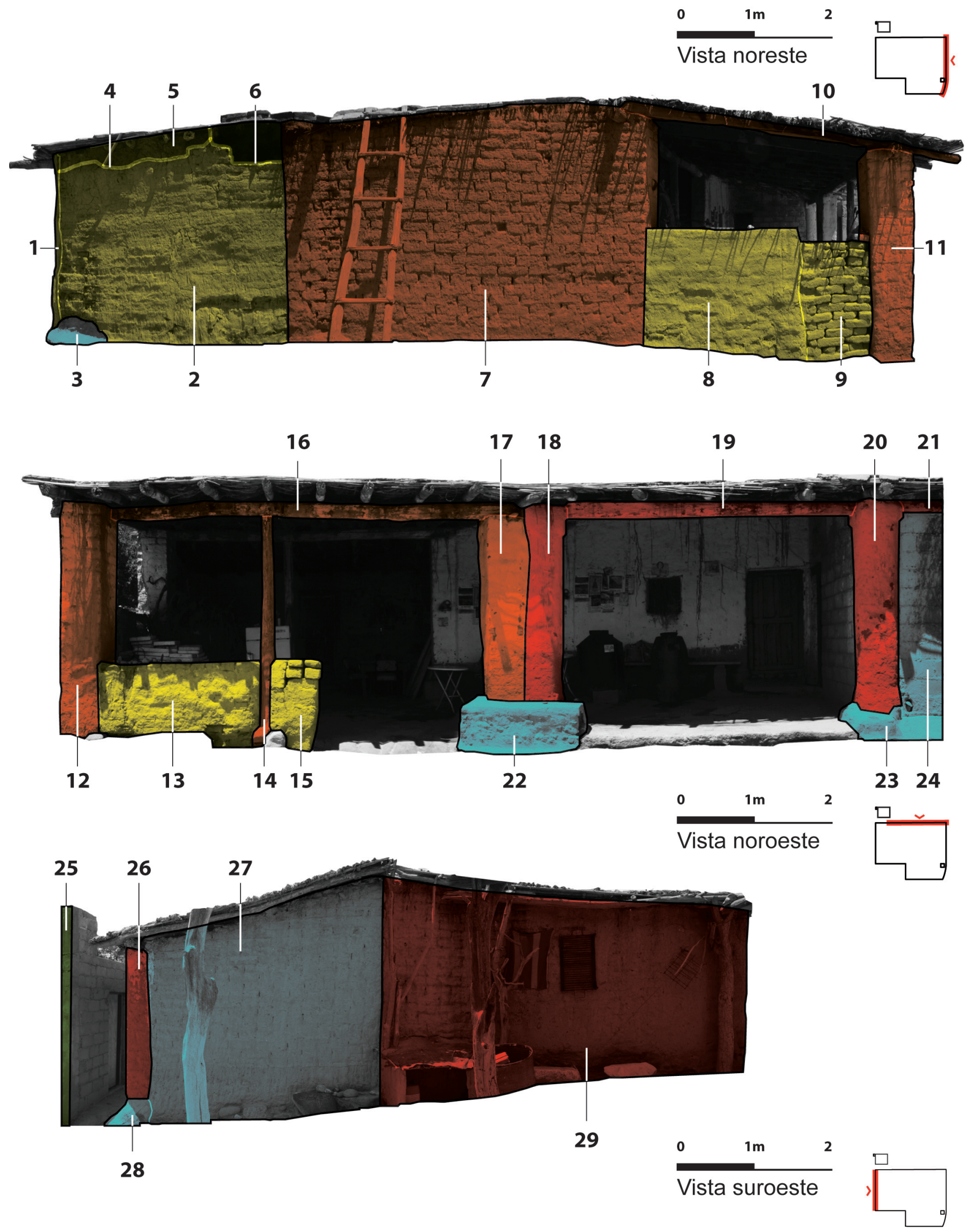

Fig. 3. Unidades estratigráficas 


\section{ANÁLISIS ESTRATIGRÁFICO DE ALZADO}
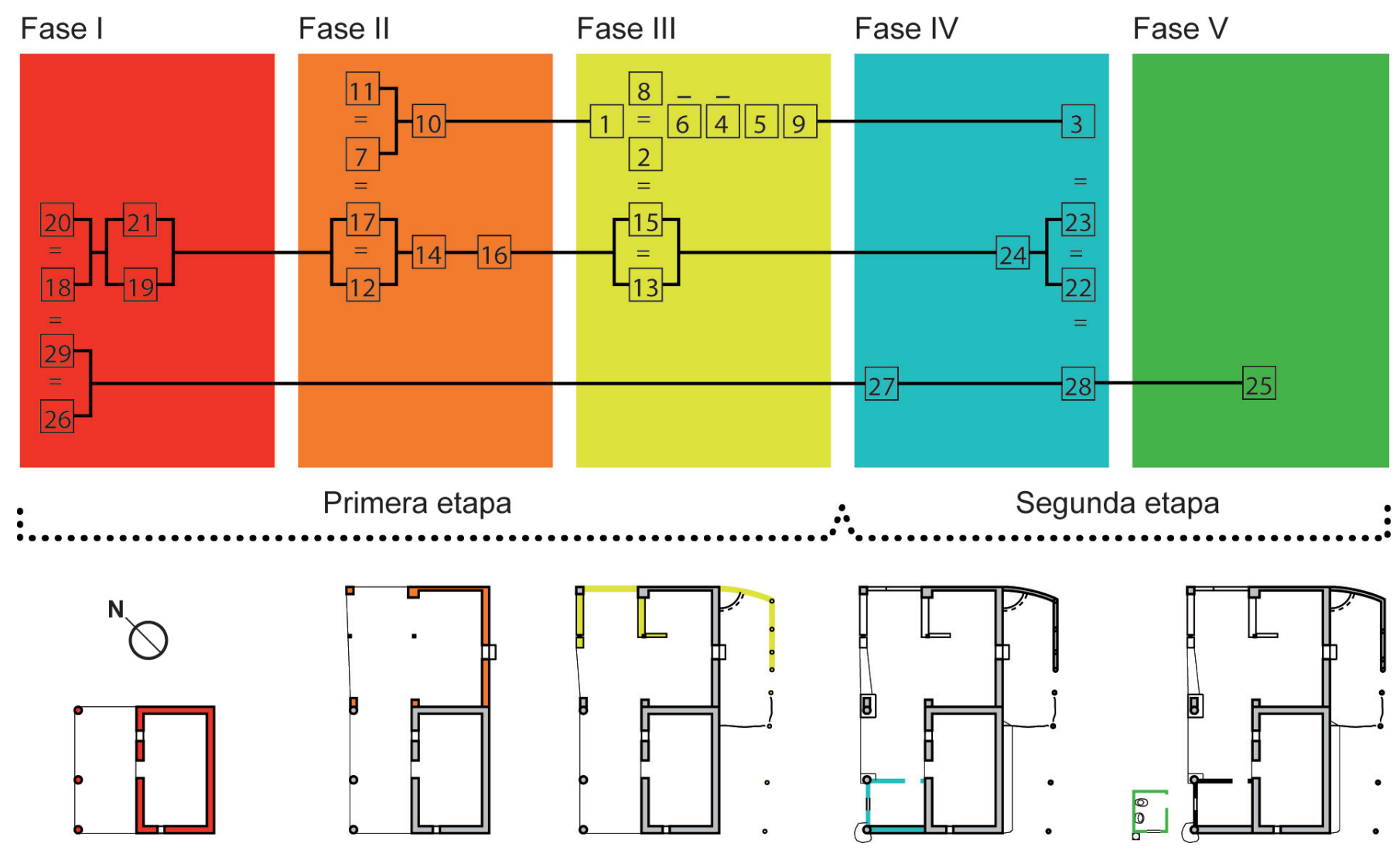

Fig. 4. Esquema estratigráfico

original, que denominamos en este caso «estar». Esta adición prolongó la galería sobre la fachada noroeste. En la ejecución de los muros se continuó empleando la albañilería de adobe; en la pared sureste, de $40 \mathrm{~cm}$ de espesor, se colocaron adobes con aparejo a tizón, mientras que en la pared noreste, de $20 \mathrm{~cm}$ de espesor, fueron dispuestos con aparejo a soga. Se construyeron cuatro columnas cuadradas de albañilería de adobe; tres de ellas tienen dimensiones de $40 \times 40 \mathrm{~cm}$ y una es de $60 \times 60 \mathrm{~cm}$. Dos de estas columnas se colocaron adosadas a la construcción preexistente, otra está libre y una cuarta se dispuso formando aparejo con uno de los muros nuevos. Como no se construyó pared central, en su lugar se colocó un rollizo de algarrobo como viga cumbrera del techo. Se colocaron dos puntales de madera en la parte media de la viga cumbrera y de la viga de borde para reducir el esfuerzo de flexión. La disposición del techo y de los cabios se mantuvo como en la unidad original, pero se omitió la colocación del cielorraso de cañas quedando expuesta la capa de enramada.
Fase III

Se amplió sobre la pared sureste un sector donde empezó a funcionar un fogón. Esta habitación se emplea actualmente para la preparación diaria de alimentos y como comedor cuando son pocas personas. Los adobes de los muros se dispusieron en aparejo a soga. Es evidente el avanzado deterioro de los adobes en las caras externas del muro, posiblemente como consecuencia de una fabricación de menor calidad. Parte de esta habitación quedó abierta y cubierta precariamente con unas membranas plásticas traslucidas. La estructura de sostén del techo se armó exclusivamente con rollizos de madera y columnas de horcones ${ }^{12}$. En este caso los muros se destinaron sólo a la función de cerramiento. La cubierta está formada por una enramada, papeles de diario y torta. Son coetáneas las elevaciones de pequeños muros al interior del estar que redujeron sus dimensiones y definieron aún más el espacio

${ }^{12}$ Rollizo de madera vertical empotrado en el piso cuya parte superior termina en forma de «V» y permite recibir y estabilizar las vigas horizontales. 
de galería hacia la fachada noroeste. Al exterior también se elevan muros que contribuyen a definir mejor los límites de esta galería.

\section{Fase IV}

Se caracteriza principalmente por la introducción de nuevos materiales: muros de bloques de cemento y cerramientos industrializados. Se dispuso un dormitorio en el extremo suroeste de la galería, donde uno de los muros elevados empleó muro de adobes y los otros dos muros son de bloques de cemento de $38 \times 19 \times 13 \mathrm{~cm}$, asentados sobre pequeñas vigas de hormigón. La puerta y la ventana son de marcos de chapa doblada. Se efectúan algunos refuerzos inferiores en los muros y recalces de las columnas con morteros de suelo-cemento.

\section{Fase $\mathrm{V}$}

Corresponde a una construcción separada del conjunto del edificio destinada a baño. Fue ejecutada por un plan nacional de vivienda para la construcción de núcleos húmedos en viviendas precarias. Empleó sólo materiales industrializados: bloques de cemento para muros y techo, cerramientos metálicos para puerta y ventana, piezas de loza cerámica para inodoros y lavabo, cañerías de plástico y una pequeña caldera metálica externa para reserva de agua caliente.

\section{LA VIVIENDA: CONSTRUCCIÓN ESTRATIFICADA Y TRANSICIÓN}

En general, de las relaciones de antero-posterioridad se detectaron al menos cinco fases, en las tres primeras (primera etapa) se emplearon la tecnología de construcción con tierra, mientras que en las dos siguientes (segunda etapa) se evidenció un cambio en la resolución constructiva debido a la introducción y predominio de nuevos materiales y técnicas.

Desde una perspectiva tecnológica, entre las fases de la primera etapa se observó un proceso de pauperización gradual de las técnicas constructivas y de algunas características morfológicas: a). Los adobes de la fase III presentan un deterioro más pronunciado que los adobes correspondientes a las fases I y II. b). Los muros de la fase III dejan de emplearse como portantes y se abandona la ortogonalidad en la unión de los mismos. c). En la fase II y III comienzan a emplearse muros de menor espesor $(20 \mathrm{~cm})$ colocados con aparejo a soga. d). No se aprecia mantenimiento periódico de los revoques externos, en la actualidad casi totalmente inexistentes por la acción erosiva del clima y la falta de mantenimiento periódico. e). El tipo de cielorraso de la fase I no vuelve a emplearse en las dos siguientes fases (se abandona el uso de la caña como terminación de superficie). f). El tipo morfológico de columna circular de la fase I deja de emplearse en las siguientes fases.

Los muros que emplean tecnología de construcción con tierra, si bien son albañilerías que no se deterioran con facilidad, resultan sensibles a la acción de la intemperie cuando media en ellos la acción prolongada del paso del tiempo y la falta de mantenimiento (Maldonado Ramos y Vela Cossío, 1999: 5). Por lo tanto, las variaciones en las técnicas de producción de los mismos se verán refejados en estados más o menos avanzados de deterioro de éstos (siempre y cuando no reciban un mantenimiento periódico o transiten por largos estados de abandono). De este modo, el deterioro es un factor clave a tener en cuenta al momento de abordar el estudio estratigráfico de este tipo de construcciones, de la misma manera que lo pueden ser el análisis granulométrico, el aparejo, el contenido de fibra vegetal o el color. Este aspecto diferencia a las albañilerías de tierra de otros tipos de muros donde se emplean la piedra o el ladrillo como materiales de construcción.

En la segunda etapa se presentó una situación radicalmente distinta, consecuencia directa del cambio de materiales y de técnicas constructivas. Se comenzó a utilizar bloques de hormigón para la construcción introduciendo modificaciones en el sistema modular empleado hasta el momento. Se redujo nuevamente el espesor del muro, ahora a $13 \mathrm{~cm}$, y se dispusieron aberturas industrializadas con medidas preestablecidas que están en relación con la dimensión de los bloques empleados. Tecnológicamente, la fase $\mathrm{V}$ fue continuación de la fase IV, pero en este caso se empleó el sistema constructivo en forma completa con predominio de materiales industrializados y con el uso de hormigón para los elementos constructivos y estructurales.

De la lectura funcional se observó que las características espaciales de la vivienda también sufrieron transformaciones progresivas. En la fase II, la yuxtaposición de un nuevo espacio semicerrado a la unidad original alteró las características funcionales de la galería. Este evento, vinculado a un claro aumento en la composición de la familia, probablemente permitió alojar gran parte de los usos diarios que se desarrollaron inicialmente en la galería, en un espacio más amplio y cómodo. Esto suceso provocó, además, un incremento en las dimensiones del espacio de galería sobre la fachada noroeste de la vivienda (Fig. 4, Fase II) que terminó de concretarse como tal durante la fase posterior con la incorporación de diversos muros. Durante la fase III se incorporó un nuevo espacio donde se ubicaron 
un fogón para calefacción, un espacio de cocina y un pequeño comedor. Estas actividades se desarrollaban previamente en la galería de la fase I y luego en el estar de fase II junto con otras actividades diarias, adquiriendo, de este modo, un espacio propio. En la fase IV se cerró un sector de la galería original para destinarla a habitación. La ocupación de la galería podría interpretarse como la materialización de dos procesos que implicaron la transición de su uso como un espacio social hacia otro meramente circulatorio y el incremento, en número y especialización funcional, de estos espacios. Este proceso de especialización de los espacios domésticos en el transcurso de estas tres fases (II a IV) continuó en la fase V. La nueva construcción alberga una nueva función, la de baño, que si bien no es una función que estuviese incorporada previamente en la vivienda, es un hecho más que acentuó este proceso de especialización mencionado. Por otro lado, las incorporaciones del sector de cocina de la fase III y del baño de la fase $\mathrm{V}$ introdujeron alteraciones a la tipología lineal de esta vivienda en dirección a un tipo en «L» o en «Z» todavía no muy claramente establecido.

Con la individualización de las unidades estratigráficas, la caracterización de las técnicas constructivas y la identificación de los materiales empleados en cada fase, fue posible establecer una cronología relativa, herramienta de análisis útil para abordar el estudio de la vivienda como un proceso, como una estructura real (Caballero Zoreda, 2009) y no como un modelo estático dado.

En cuanto al estudio tipológico, Caballero Zoreda (Ibidem) sostiene que los tipos son factibles de ser empleados como indicadores cronológicos absolutos. En este sentido, es importante la aportación de Armellini et al (1970: 23) al establecer una cronología para la vivienda del valle de Antinaco-Los Colorados en La Rioja. En la relación cronológica de antero-posterioridad de nuestro estudio, se concluye que el edificio histórico contiene a la unidad original dentro de la estructura general de la vivienda (Fig. 5) y se corresponde con el modelo descripto para el subtipo Vivienda de transición ${ }^{13}$ de esta región de valles riojanos (Ibidem: 10). Este dato nos aportó elementos fundamentales para confirmar que el momento de construcción de la vivienda estudiada correspondería a mediados del siglo XIX. Por otro lado, la vivienda en estudio "transita", desde su origen hasta la actualidad, por diversas fases posteriores. Las transformaciones de cada fase no provocaron cambios sustanciales en el subtipo

\footnotetext{
${ }^{13}$ Se refiere al subtipo intermedio entre los subtipos Rancho y Casa Criolla I de la respectiva clasificación.
}
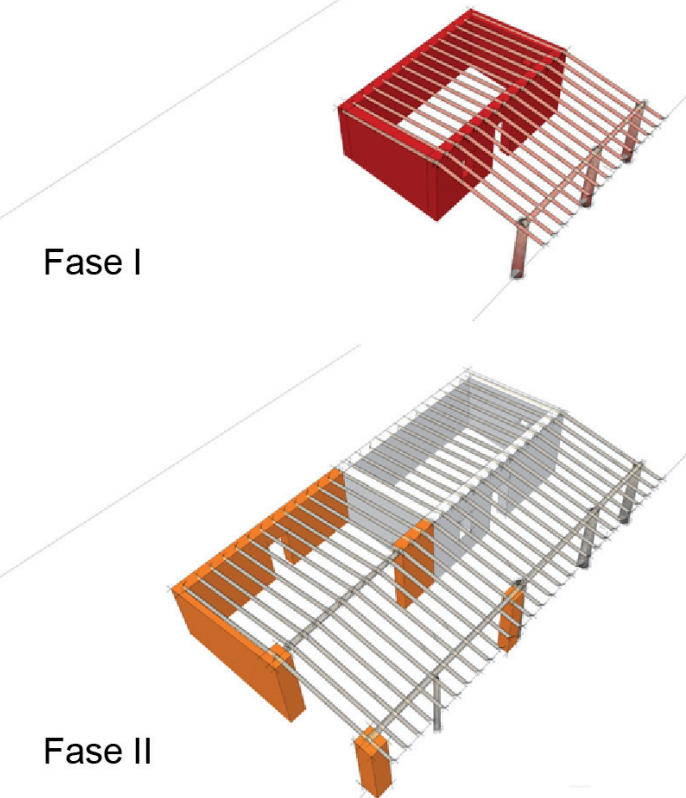

Fase III
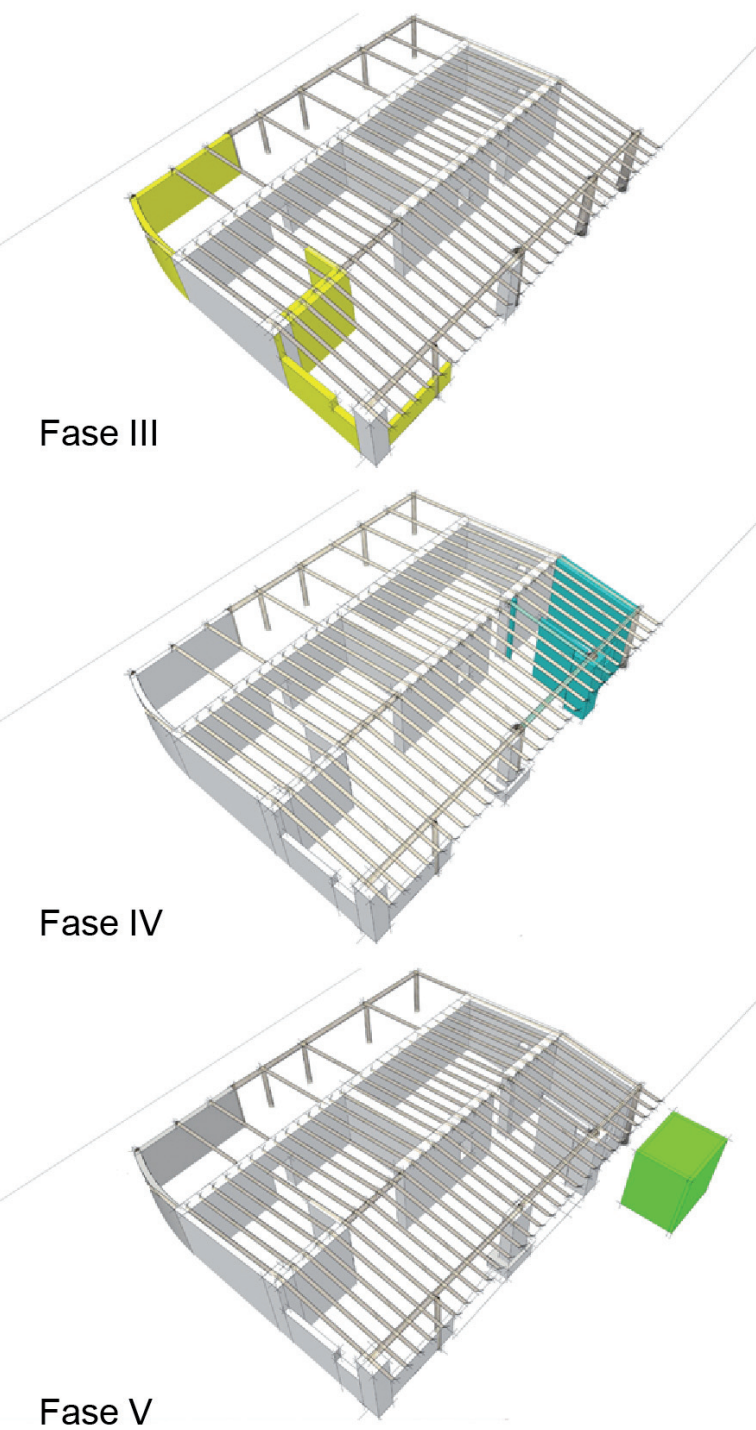

Fig. 5. Esquema de crecimiento. Imagen elaborada por Mariano Ledesma 
establecido al que se ajusta el edificio, salvo por la introducción de nuevos materiales que es característico de otro subtipo de la misma clasificación (Ibídem: 20). Sin embargo, identificar el tipo permitió una mejor comprensión de los cambios funcionales, constructivos y morfológicos que ocurrieron en cada etapa. Es en este punto donde consideramos que la complementariedad con la estratigrafía de alzados permite avanzar más en la interpretación, aportando un volumen de información importante sobre los procesos técnicos y las transformaciones espaciales que tuvieron lugar, aspectos que el estudio tipológico por si sólo no podría aportar. La Casa Zulma Castro es un ejemplo de vivienda de transición, pero no porque encaje en un tipo en particular, sino por la naturaleza misma de edificio histórico.

\section{CONCLUSIONES}

El análisis estratigráfico de alzado abrió un panorama amplio a este estudio tanto para el análisis como para la interpretación del proceso evolutivo de esta vivienda al poder individualizar y ordenar un número importante de hechos constructivos. De la misma manera, el análisis tipológico de la vivienda rural contribuyó al análisis estratigráfico, en especial cuando fue asociado al carácter funcional de los espacios y su relación entre ellos. Por otro lado, al vincular ambos análisis quedó al descubierto, dentro del conjunto actual del edificio, la unidad original como un modelo sencillo y completo del tipo de vivienda rural popular, característico del siglo XIX en la región de valles de La Rioja.

En referencia a las técnicas constructivas, se observó un proceso dinámico que transcurrió desde el empleo determinante de la tierra como parte de una tradición constructiva vernácula hasta la aceptación y preferencia de materiales industrializados. Esto nos permite señalar que, en este caso específico, tuvo lugar un proceso que implicó la pérdida gradual de las reglas del arte de construir con tierra en torno a este edificio en favor de una preferencia abrupta por emplear materiales parcial o totalmente elaborados industrialmente. Este es un caso donde los diversos factores, a los que hacía referencia Cirvini (2005), ponen en riesgo la continuidad de las tradiciones constructivas vernáculas en tierra del área de valles de La Rioja.

Finalmente, el proceso que resulta de habitar la vivienda dio lugar a distintos y sucesivos edificios (Mañana Borrazás, 2002; Caballero Zoreda, 2009) en su transcurso por diversos estadíos de reformulación y especialización de los espacios domésticos que, en este caso de estudio, se pudieron identificar a partir de ampliaciones espaciales. Si bien las herramientas de análisis de la AA permitieron identificar las modificaciones en esta vivienda rural a partir de factores tecnológicos, formales y funcionales, en coincidencia con lo que plantea Azkarate (2002: 57), creemos que esta disciplina puede contribuir de manera categórica propiciando una lectura más profunda de los contextos sociales y productivos de donde surgen y pertenecen las edificaciones y sus productores.

\section{BIBLIOGRAFÍA}

AA. VV. 1987. Arquitecturas de tierra cruda y de tierra cocida. Revista Summa, colección temática No 19. Buenos Aires.

Aparicio, F. de. 1931. La vivienda natural en la provincia de Córdoba. Publicación del Museo antropológico y etnográfico de la Facultad de Filosofía y Letras. Buenos Aires.

Aparicio, F. de. 1937. La vivienda natural en la provincia de La Rioja. Noticia preliminar. Anales de la Sociedad Argentina de Estudios Geográficos. Tomo V: 429-433. Buenos Aires.

Ardissone, R. 1948. Contribución al estudio de la vivienda argentina. Revista Humanidades. Tomo 31: 65-104. Buenos Aires.

Armellini, O., Cóppola, H., Iglesias Molli, G., Rosso, R. 1970. Anexo 3.1: Estudio particularizado de la vivienda en el área. Instituto de Investigaciones de la Vivienda. Programación de vivienda y servicios comunitarios en el Valle de Antinaco - Los Colorados: Provincia de La Rioja. Buenos Aires.

Azkarate Garai-Olaun, A. 2002. Intereses cognoscitivos y praxis social en Arqueología de la Arquitectura. Arqueología de la Arquitectura, 1: 55-71.

Bardou, P. y Arzoumanian, V. 1979. Arquitecturas de adobe. Editorial Gustavo Gili. Barcelona. España

Caballero Zoreda, L. 2009. Edificios históricos y Arqueología: un compromiso entre exigencias, responsabilidad y formación. Arqueología de la Arquitectura, 6: 11-19.

Cáceres Freyre, J. 1946. En torno al estudio de la vivienda rural argentina. Anales de la Asociación Folklórica Argentina. Volumen II: 91-93. Buenos Aires.

Calla García, A. 1999. Red Habiterra: Sistematización del uso de la tierra en viviendas de interés social. El Habitat Iberoamericano en la mira. El Habitat en CYTED se llama HABYTED: 35-44. Subprograma XIV Viviendas de Interés Social. Asunción, Paraguay.

Canepuccia, P., Castro, H., Ocvirk, M., Ostropolsky, E. 1976. Viviendas tradicionales en zona árida: La Rioja. Programa de la OEA para la Vivienda. Publicación del centro de investigación Mendoza - IADIZA - LAHV. Mendoza. Argentina

Chiozza, E. 1945. Vivienda natural en Tolombóm (Provincia de Salta, República Argentina). Actes du XXVIIIe Congrès international des américanistes de Paris: 109-114. París.

Cirvini, S. 2005. Los valores y significados del Patrimonio Vernáculo en Tierra. Su relación con la conservación y con la construcción de nuevas obras en la región de Cuyo - Argentina. CD del V SIACOT. Mendoza. Argentina.

De Hoz Onrubia, J., Maldonado Ramos, L. y Vela Cossío, F. 2003. Diccionario de construcción tradicional: tierra. Editoria Nerea, San Sebastián. España.

Dethier, J. 1982. Des Architectures de terre. Ou l'avenir d'une tradition millénaire. Centre National d'Art et de Culture Georges Pompidou. Paris.

Fathy, H. 1973. Architecture for the poor: an experiment in rural Egypt. Chicago, Estados Unidos.

Instituto de Investigaciones de la Vivienda. 1969. Tipos predominantes de vivienda natural en la República Argentina. FAU - UBA. Buenos Aires.

Jiménez Delgado, M., Cañas Guerrero, I. 2007. The selection of soils for unstabilised earth building: A normative review. Construction and Building Materials, 21: 237-251

Houben, H., Guillaud, H. 1994. Earth building: a comprehensive guide. CRA Terre-EAG, Greenbelt, Francia.

Maldonado Ramos, L. y Vela Cossío, F. 1999. Curso de Construcción con Tierra I. Técnicas y sistemas tradicionales. Escuela Técnica Superior de Arquitectura de Madrid. Madrid. 
Maldonado Ramos, L. y Rivera Gámez, D. 2002. La arquitectura construida con tierra en el umbral del siglo XXI. En: Arquitectura y Construcción con Tierra. Tradición e Innovación, pp. 67-82. Madrid.

Maldonado Ramos, L., Rivera Gámez, D. y Vela Cossío, F. 2002. La protección del patrimonio edificado con tierra. Instrumentos de gestión, difusión e investigación. El Centro de Investigación de Arquitectura Tradicional. Anais I Seminario Ibero-americano de Construçao com Terra, pp. 177-198. Salvador de Bahía, Brasil.

Mañana Borrazás, P., Blanco Rotea, R., Ayán Villa, X. 2002. Arqueotectura 1: Bases Teórico Metodológicas para una Arqueología de la Arquitectura. Traballos de Arqueoloxia e Patrimonio (TAPA) 25. España.

Martínez-Camacho, F., Vazquez-Negrete, J., Lima, E., Lara, V., Bosch, P. 2008. Texture of nopal treated adobe: restoring Nuestra Señora del Pilar mission. Journal of Archaeological Science, 35: 1125-1133.

Morel, J., Mesbah, A., Oggero, M., Walker, P. 2001. Building houses with local materials: means to dastically reduce the environmental impact of construction. Building and Environment, 36 (10): 1119-26.

Neves, C. 2004. Proyecto 6 PROTERRA/CYTED. Seminario Internacional de Construcción con Tierra. CYTED/HABYTED-PROTERRA. Memorias en CD-RM. El Salvador.

Parenti, R. 1996. Individualización de las unidades estratigráficas murarias. Arqueología de la Arquitectura. Burgos, pp. 75-85. España.

Rotondaro, R. y Kirschbaum, C. 1993. Appropriated earth roofs for the altiplano of Argentina. $7^{\text {th }}$ Conferencia Internacional Sobre o Estudo e Conservacao da Arquitectura de Terra-TERRA93: 393-397. DGEMN- ICCROM- CRATerre - ICOMOS. Portugal.

Rotondaro, R. 2007. Arquitectura de Tierra contemporánea: tendencias y desafíos. Revista Apuntes, Vol. 20, 2: 342-353.
Santos Pinheiro, N. 1993. Terra. Material milenario de construçao. $7^{a}$ Conferencia Internacional sobre o Estudo e Conservaçao da Arquitectura de Terra "Terra93", pp. 103-107. Lisboa, Portugal.

Viñuales, G. 1981. Restauración de Arquitectura de Tierra. Instituto Argentino de Investigaciones de Historia de la Arquitectura y del Urbanismo. Tucumán, Argentina.

Viñuales, G. 1987. Diseño, historia y tecnología de las arquitecturas de tierra cruda. Arquitecturas de tierra cruda y de tierra cocida. Summa No19 Colección temática: 9-19. Buenos Aires.

Viñuales, G. 1990. Construcción tradicional en el nordeste argentino. 6th International Conference on the Conservation of Earthen Architecture "Adobe 90», pp. 137-140. New Mexico, Estados Unidos.

Viñuales, G. 1991. La arquitectura en tierra en la región andina. Anales del Instituto de Arte Americano e Investigaciones Estéticas, 27-28: 43-53. Buenos Aires.

Viñuales, G., Martins Neves, C., Flores, M., Ríos, M. 1994. Arquitecturas de Tierra en Iberoamérica. Habiterra. Programa de Ciencia y Tecnología para el Desarrollo, CYTED. Buenos Aires.

Tiwari, P., Parikh, K., Sharma, V. 1996. Performance evaluation of cost effective building - A cost, emissions employment point of view. Building and Environment, 31(1): 75-90.

Zamorano, M. 1950. Acerca de la vivienda natural en la República Argentina y especialmente en Mendoza. Anales de arqueología y etnología. Tomo 11: 89110. Buenos Aires.

Recibido: 7 de julio de 2010 Aceptado: 21 de octubre de 2010 